\title{
Remark on the Normal Forms of Diversors of Second Order Differential Equations of Normal Hyperbolic Type
}

\author{
M. Burkhardt
}

Dedicated to the memory of Professor Paul Günther (1926 - 1996)

\begin{abstract}
With respect to the monograph of P. Günther "Huygens' Principle and Hyperbolic Equations" this paper contains a supplement to diversors of second order differential equations of normal hyperbolic type [3: Chapter IV]. We construct a "normal form" of a diversor and consider the coefficients of this form in a certain neighbourhood of the characteristic backward conoid $C_{-}(\xi)$ of a point $\xi$.
\end{abstract}

Keywords: Diversors, Riesz distributions

AMS subject classification: $35 \mathrm{~L} 10,35$ A 30, 35 A 08, 58 G 16

Let $(M, g)$ be a pseudo-Riemannian manifold with finite dimension $m=\operatorname{dim} M>2$ whose metric $g$ has Lorentz signature $\{+,-, \ldots,-\}$. It is always assumed that $M$ is of class $C^{\infty}$, connected and satisfying the second axiom of countability; $g$ is of class $C^{\infty}$ on $M . \nabla$ denotes the Levi-Civita connection of $(M, g)$.

Let $\Omega \subseteq M$ be a geodesically normal domain and $\Omega_{0} \subseteq \Omega$ any causal domain in $\Omega$ (see also [3: p. 15]). We consider any domain $\Omega$ and choose in $\Omega$ any coordinate system $\rho: \Omega \rightarrow \mathbb{R}^{m}$, where $\Omega \subseteq M$ is open. We denote the second order differential operator of normal hyperbolic typc of $(M, g)$, acting on scalar functions $u$, by $P$ :

$$
\begin{aligned}
P[u] & =g^{i j} \nabla_{i} \nabla_{j} u+A^{i} \nabla_{i} u+C u \\
& =\frac{1}{\sqrt{g}} \frac{\partial}{\partial x^{i}}\left(\sqrt{g} g^{i j} \frac{\partial u}{\partial x^{j}}\right)+A^{i} \frac{\partial u}{\partial x^{i}}+C u \quad(i, j=1,2, \ldots, m)
\end{aligned}
$$

and the invariant measure associated to the metric $g$ by $\mu$ which is given in these coordinates $\left\{x^{1}, \ldots x^{m}\right\}$ by

$$
\mu=\sqrt{g} d x^{1} \wedge \ldots \wedge d x^{m}
$$

Let the point $\xi \in \Omega$ be fixed. We denote the characteristic conoid by $C(\xi)$ given by the equation $\Gamma(\xi, x)=0$ where $\Gamma(\xi, x)$ is the quadratic geodesic distance function.

M. Burkhardt: Math. Institut der Universität, Augustuspl. 10/11, D - 04109 Léeipzig 
The notion "diversor" is due to L. Asgeirsson [1]. He defines a diversor $D$ of $P$ as a differential operator $D$, such that $D \circ P$ can be written as divergence expression on the characteristic conoid $C(\xi)$, the vertex $\xi$ excluded.

Now we consider the characteristic backward conoid $C_{-}(\xi)$. Let $\Omega_{0}^{\prime} \subseteq \Omega_{0}$ be a domain such that $C(\xi) \cap \Omega_{0}^{\prime}=\left(C_{-}(\xi) \backslash\{\xi\}\right) \cap \Omega_{0}$, i. e. the vertex $\xi \notin \Omega_{0}^{\prime}$.

Definition 1. Let $\phi \in C_{0}^{\infty}\left(\Omega_{0}^{\prime}\right)$ be any test function. A differential operator $D$ is said to be a diversor of $P$ with respect to $C_{-}(\xi)$ if

$$
\int_{C_{-}(\xi)}(D \circ P)[\phi](x) \nu(x)=0
$$

i.e. the distribution $v \in \mathcal{D}^{\prime}\left(\Omega_{0}^{\prime}\right)$ with

$$
(v, \phi)=\int_{C_{-}(\xi)} D[\phi](x) \nu(x)
$$

is a solution of $P^{*}[v]=0$ in $\Omega_{0}^{\prime}$ with supp $v \subseteq C_{-}(\xi) \backslash\{\xi\}$ where $\nu(x)$ denotes the Leray form of the submanifold $C_{-}(\xi)$ (see also [3: Chapter II, §2]), $P^{*}$ denotes the (invariantly) formally adjoint operator of $P$.

Such an identity (2) is only possible if $(D \circ P)[\phi]$ can be written in divergence form with respect to the submanifold $C_{-}(\xi)$ in $\Omega_{0}^{\prime}$.

Definition 2. Two diversors $D_{1}$ and $D_{2}$ in $\Omega_{0}^{\prime}$ are called equivalent if

$$
\int_{C_{-}(\xi)} \dot{D}_{1}[\phi](x) \nu(x)=\int_{C_{-}(\xi)} D_{2}[\phi](x) \nu(x) \quad\left(\phi \in C_{0}^{\infty}\left(\Omega_{0}^{\prime}\right)\right)
$$

i.e. $\left.D_{1}[\phi]-D_{2} \mid \phi\right]$ is a divergence expression on the characteristic semiconoid $C_{-}(\xi)$.

Proposition 1. Let $\left\{\bar{x}^{1}, \bar{x}^{2}, \ldots, \bar{x}^{m}\right\}$ be a local coordinate system in $\Omega_{0}^{\prime}$, such that $C_{-}(\xi)$ is given by $\bar{x}^{1}=0$, i.e.

$$
\left.\begin{array}{l}
\bar{x}^{1}=\Gamma(\xi, x) \\
\bar{x}^{\alpha}=x^{\alpha} \quad(\alpha=2,3, \ldots, m)
\end{array}\right\}
$$

For each diversor $D$ there exists an equivalent linear differential operator which is called $D_{N}$ of the form

$$
D_{N}[\phi]=\frac{1}{\sqrt{g}} \sum_{\nu=0}^{\kappa} \frac{\partial^{\nu}}{\partial \Gamma^{\nu}}\left(w_{\kappa-\nu} \cdot \phi\right)
$$

The coefficients $w_{\kappa-\nu}$ are of class $C^{\infty}$ in $\Omega_{0}^{\prime}$ and are uniquely determined on $C_{-}(\xi)$. The form (4) of a diversor is said to be normal form $D_{N}$ of $D$ of order $\kappa$.

Proof. The proof is obvious. The derivates of highest order in $D[\phi]$ are not all interior derivates $\partial^{\alpha} / \partial \bar{x}^{\alpha}$ with respect to the manifold $\bar{x}^{1}=0$, consequently, the order of $D$ cannot be reduced with the help of integration by parts [3: pp. 270,271] 
Proposition 2. To each diversor $D$ of order $\kappa$ of $P$ with respect to $C_{-}(\xi)$ there exists an equivalent diversor in normal form (4) whose "modified coefficients" $W_{\nu}$ with

$$
W_{\nu}:=\frac{\partial_{1} \Gamma(\xi, x)}{\sqrt{g}} w_{\nu} \quad(\nu=0,1,2, \ldots \kappa)
$$

in $\Omega_{0}^{\prime}$ are given by

$$
\begin{aligned}
g^{i j} \nabla_{i} \Gamma \nabla_{j} W_{0}+\left(M^{*}+n-4-2 \kappa\right) W_{0} & =0 \\
g^{i j} \nabla_{i} \Gamma \nabla_{j} W_{\nu}+\left(M^{*}+n-4-2 \kappa+2 \nu\right) W_{\nu} & =\frac{1}{2} P^{*}\left[W_{\nu-1}\right] \quad(\nu=1,2, \ldots, \kappa) \\
L^{*}\left[W_{\kappa}\right] & =0 \text { on } C_{-}(\xi)
\end{aligned}
$$

with

$$
M^{*}(\xi, x)=\frac{1}{2} g^{i j} \nabla_{i} \nabla_{j} \Gamma-\frac{1}{2} A^{i} \nabla_{i} \Gamma-n .
$$

Proof. Let $\Omega_{0}^{\prime \prime} \subseteq \Omega_{0}^{\prime}$ be a neighbourhood of $C_{-}(\xi)$ with the condition $\partial_{1} \Gamma \neq 0$. $\left(\Delta_{2}=g^{i j} \nabla_{i} \nabla_{j}\right.$ denotes the 2. Beltrami operator.) In $\Omega_{0}^{\prime \prime}$ we obtain by the (regular) transformation to the coordinates $\bar{x}^{i}$

$$
\begin{gathered}
\bar{g}^{11}=4 \Gamma, \quad \bar{g}^{1 \beta}=g^{i \beta} \partial_{i} \Gamma, \quad \bar{g}^{\alpha 1}=g^{\alpha j} \partial_{j} \Gamma, \quad \bar{g}^{\alpha \beta}=g^{\alpha \beta} \\
\sqrt{g}=\left|\partial_{1} \Gamma\right| \sqrt{\bar{g}}, \quad \sqrt{\bar{g}}=\frac{\sqrt{g}}{\left|\partial_{1} \Gamma\right|} \\
\bar{\Gamma}^{1}=-\Delta_{2} \Gamma, \quad \bar{\Gamma}^{\alpha}=\Gamma^{\alpha} \quad\left(\Gamma^{i}=g^{k j} \Gamma_{k j}^{i}\right) \\
\bar{A}^{1}=A^{i} \nabla_{i} \Gamma, \quad \bar{A}^{\alpha}=A^{\alpha} \\
\frac{\partial}{\partial \bar{x}^{1}}=\frac{1}{\left|\partial_{1} \Gamma\right|} \frac{\partial}{\partial x^{1}}, \quad \frac{\partial}{\partial \bar{x}^{\alpha}}=-\frac{\partial_{\alpha}}{\left|\partial_{1} \Gamma\right|} \frac{\partial}{\partial x^{1}}+\frac{\partial}{\partial x^{\alpha}} \\
\bar{g}^{1 j} \frac{\partial}{\partial \bar{x}^{j}}=g^{i j} \nabla_{i} \Gamma \nabla_{j}
\end{gathered}
$$

and by explicit calculations the expression

$$
\begin{aligned}
D \circ P[\phi]= & \frac{1}{\sqrt{g}} \sum_{\nu=0}^{\kappa} \frac{\partial^{\nu}}{\partial \Gamma^{\nu}}\left(w_{\kappa-\nu} \cdot P[\phi]\right) \\
= & \frac{1}{\sqrt{g}} \cdot \operatorname{Div}[\phi] \\
& +\frac{1}{\sqrt{g}} \phi\left[P^{0}[\phi]+\sum_{\nu=1}^{\kappa+1} \frac{\partial^{\nu}}{\partial \Gamma^{\nu}}\left(P^{0}\left[w_{\kappa-\nu}\right]+(N+4 \nu+4)\left[w_{\kappa-\nu+1}\right]\right)\right] \\
& +\frac{1}{\sqrt{g}} \sum_{r=1}^{\kappa} \frac{\partial^{r} \phi}{\partial \Gamma^{r}}\left[\sum_{\nu=r}^{\kappa+1}\left(\begin{array}{l}
\nu \\
r
\end{array}\right) \frac{\partial^{\nu-r}}{\partial \Gamma^{\nu-r}}\left(P^{0}\left[w_{\kappa-\nu}\right]+(N+4 \nu+4)\left[w_{\kappa-\nu+1}\right]\right)\right] \\
& +\frac{1}{\sqrt{g}} \frac{\partial^{\kappa+1} \phi}{\partial \Gamma^{\kappa+1}} \cdot(N+4 \kappa+8)\left[w_{0}\right]
\end{aligned}
$$


with

$$
\begin{aligned}
N[\phi]: & =-2 \frac{\partial\left(\bar{g}^{1 j} \phi\right)}{\partial \bar{x}^{j}}+\left(\bar{A}^{1}-\bar{\Gamma}^{\mathbf{l}}\right) \phi \\
& =-2 \frac{\sqrt{g}}{\left|\partial_{1} \Gamma\right|} g^{i j} \nabla_{i} \Gamma \nabla_{j}\left(\frac{\left|\partial_{1} \Gamma\right|}{\sqrt{g}} \phi\right)+\left(-\Delta_{2} \Gamma+\left(\nabla_{i} \Gamma\right) A^{i}\right) \phi \\
(N+k)[\phi]: & =N[\phi]+k \phi \quad(k \in \mathbb{N}) \\
P^{0}[\phi]: & =\sqrt{\bar{g}} P^{*}\left[\frac{\phi}{\sqrt{g}}\right]=\frac{\sqrt{g}}{\left|\partial_{1} \Gamma\right|} P^{*}\left[\frac{\left|\partial_{1} \Gamma\right|}{\sqrt{g}} \phi\right] .
\end{aligned}
$$

Because (2) we obtain that the coefficient $w_{0}$ satisfies the equation

$$
(N+4 \kappa+8)\left[w_{0}\right]=0
$$

at first on $C_{-}(\xi)$. Now $(10)$ (and $w_{0}$ ) can be extended to $\Omega_{0}^{\prime}$. (It is a transition to an equivalent diversor.) Successively, in $\Omega_{0}^{\prime}$ we obtain that the coefficients $w_{1}, w_{2}, \ldots, w_{\kappa}$ are solutions of

$$
(N+4 \kappa-4 \nu+8)=-P^{0}\left[w_{\nu-1}\right]
$$

and, finally,

$$
P^{0}\left(w_{\kappa}\right)=0 \quad \text { on } C_{-}(\xi)
$$

Consequently, from (10), (11), (12) and with respect to (7), (8), (9) the assertion follows

In the case of order $\kappa=\frac{n-4}{2}$ a comparision of (6) with the equations for the Hadamard coefficients $V_{\nu}$ of the Riesz distributions (see also $[5,7,8]$ )

$$
\begin{aligned}
g^{i j} \nabla_{i} \Gamma \nabla_{j} W_{0}+M^{*} V_{0} & =0 \\
g^{i j} \nabla_{i} \Gamma \nabla_{j} W_{\nu}+\left(M^{*}+2 \nu\right) V_{\nu} & =-P^{*}\left[W_{\nu-1}\right] \quad(\nu=1,2, \ldots)
\end{aligned}
$$

shows the relations

$$
W_{\nu}(\xi, x)=(-1)^{\nu} \frac{1}{2^{\nu}} V_{\nu}(\xi, x) .
$$

Consequently, in $\Omega_{0}^{\prime \prime} \subseteq \Omega_{0}^{\prime}$ the coefficients $w_{\nu}$ are smooth.

Now we consider (2), respectively (3), but $\phi \in C_{0}^{\infty}\left(\Omega_{0}\right)$ (vertex $\xi \in \Omega_{0}$ !):

$$
\int_{C_{-}(\xi)}(D \circ P)[\phi](x) \nu(x)=0 \quad\left(\phi \in C_{0}^{\infty}\left(\Omega_{0}\right)\right)
$$

with $D$ in normal form (4) with (5) and (6). However, because these singularities of $w_{\nu}$ (for $x \rightarrow \xi$ on $C_{-}(\xi)$ ) are algebraic, it is possible to show (see [2: pp. 21, 22, 53]) that the integral (15) exists or can be regularized (in the sense of distributions). Consequently, the distribution $v \in \mathcal{D}^{\prime}\left(\Omega_{0}^{\prime}\right)$ in $(3)$ can be extended to a distribution $v \in \mathcal{D}^{\prime}\left(\Omega_{0}\right)$ over $\Omega_{0}$. Then the results about diversors in [3: Chapter IV, §3] of $P$. Günther are applicable. 


\section{References}

[1] Asgeirsson, L.: Some hints on Huygens' principle and Hadamards conjecture. Comm. Pure Appl. Math. 9 (1956), 307 - 326.

[2] Gelfand, I. M. and G. E. Schilow: Verallgemeinerte Funktionen (Distributionen). Band I. Berlin: Dt. Verlag Wiss. 1967.

[3] Günther, P.: Huygens' Principle and Hyperbolic Equations (Perspectives in Mathematics: Vol. 5). Boston: Academic Press 1988.

[4] Günther, P.: Über die Darbouxsche Differentialgleichung mit variablen Koeffizienten. Math. Nachr. 22 (1965), 285 - 321.

[5] Günther, P. and V. Wünsch: Maxwellsche Gleichungen und Huygenssches Prinzip. 'Part I. Math. Nachr. 63 (1974), $97-121$.

[6] Hadamard, J.: Lectures on Cauchy's Problem in Linear Partial Differential Equations. New Haven: Yale University Press 1923.

[7] Riesz, M.: L' intégrale de Riemann-Liouville et le probl'eme de Cauchy. Acta Math. 81 (1949), 1 - 223.

[8] Schimming, R.: Das Huygenssche Prinzip bei linearen hyperbolischen Differentialgleichungen zweiter Ordnung für allgemeine Felder. Beitr. Anal. 11 (1978), 45 - 90.

[9] Schwartz, L.: Theórie des distributions. Tome 1 et 2. Paris: Hermann 1951 - 1957. 